793 (1993); K. A. Dill et al., Protein Sci. 4, 561 (1995); J. N. Onuchic, P. G. Wolynes, Z. LutheySchulten, N. D. Socci, Proc. Natl. Acad. Sci. U.S.A 92, 3626 (1995); K. Yue et al., ibid., p. 325.

67. S. Kamtekar, J. M. Schiffer, H. Xiong, J. M. Babik, M. H. Hecht, Science 262, 1680 (1993).

68. L. Regan and N. D. Clarke, Biochemistry 29, 10878 (1990).

69. T. Handel and W. F. DeGrado, J. Am. Chem. Soc 112, 6710 (1990)

70. T. M. Handel, S. A. Williams, W. F. DeGrado, Science 261, 879 (1993)

71. D. P. Raleigh and W. F. DeGrado, J. Am. Chem. Soc. 114, 10079 (1992)

72. D. P. Raleigh, S. F. Betz, W. F. DeGrado, ibid. 117, 7558 (1995)

73. B. Honig and A.-S. Yang, Adv. Protein Chem. 46 27 (1995); K. Yue and K. A. Dill, Proc. Natl. Acad. Sci. U.S.A. 89, 4163 (1992).

74. M. Munson, R. O'Brien, J. M. Sturtevant, L. Regan, Protein Sci. 3, 2015 (1994)

75. C. E. Schafmeister, L. J. W. Miercke, R. M. Stroud, Science 262, 734 (1993).

76. D. S. Kemp and B. R. Bowen, Tetrahedron Lett. 29 5077 (1988); D. S. Kemp and B. R. Bowen, ibid., p. 5081 ; K. Y. Tsang, H. Diaz, N. Graciani, J. W. Kelly, J. Am. Chem. Soc. 116, 3988 (1994).

77. C. A. Kim and J. M. Berg, Nature 362, 267 (1993); D. L. Minor Jr. and P. S. Kim, ibid. 367, 660 (1994); ibid. 371, 264 (1994); C. K. Smith, J. M. Withka, L. Regan, Biochemistry 33, 5510 (1994); D. E. Otzen and A. R. Fersht, ibid. 34, 5718 (1995)

78. J. Richardson et al., Biophys. J. 63, 1186 (1992)

79. T. P. Quinn, N. B. Tweedy, R. W. Williams, J. S Richardson, D. C. Richardson, Proc. Natl. Acad Sci. U.S.A. 91, 8747 (1994).

80. Y. Yan and B. W. Erickson, Protein Sci. 3, 1069 (1994)

81. A. Pessi et al., Nature 362, 367 (1993); E. Bianchi, S. Venturini, A. Pessi, A. Tramontano, M. Sollazzo, J. Mol. Biol. 236, 649 (1994).

82. A. N. Fedorov et al., J. Mol. Biol. 225, 927 (1992).

83. K. Goraj, A. Renard, J. A. Martial, Protein Eng. 3 259 (1990); M. Beauregard et al., ibid. 4, 745 (1991); A. Houbrechts et al., ibid. 8, 249 (1995); T. Tanaka, Y. Kuroda, H. Kimura, S. Kidokoro, H. Nakamura, ibid. 7, 969 (1994); T. Tanaka, M. Hayashi, H. Kimura, M. Oobatake, H. Nakamura, Biophys. Chem. 50, 47 (1994); T. Tanaka et al., Protein Sci. 3, 419 (1994)

84. R. S. Farid, C. C. Moser, P. L. Dutton, Curr. Opin Struct. Biol. 3, 225 (1993).

85. C. T. Choma et al., J. Am. Chem. Soc. 116, 856 (1994)

86. D. E. Robertson et al., Nature 368, 425 (1994).

87. D. L. Huffman, thesis, University of Illinois, UrbanaChampaign (1994)

88. H. Mihara et al., Chem. Lett. 1992, 1813 (1992); G. Basu, D. Anglos, A. Kuki, Biochemistry 32, 3067 (1993); D. Anglos, V. Bindra, A. Kuki, J. Chem. Soc. Chem. Commun. 1994, 213 (1994); D. G. McCafferty et al., Tetrahedron 51, 1093 (1995); D. R. Benson, B. R. Hart, X. Zhu, M. B. Doughty, J. Am. Chem. Soc. 117, 8502 (1995).

89. M. Montal, FASEB J. 4, 2623 (1990); M. R. Ghadiri, J. R. Granja, L. K. Buehler, Nature 369, 301 (1994)

90. P. K. Kienker, W. F. DeGrado, J. D. Lear, Proc. Nat/ Acad. Sci. U.S.A. 91, 4859 (1994)

91. B. Gutte, M. Däumingen, E. Wittschieber, Nature 281, 650 (1979); W. F. DeGrado, ibid. 365, 488 (1993).

92. K. Johnsson, R. K. Allemann, H. Widmer, S. A Benner, ibid. 365 , p. 530 .

93. S. E. Blondelle, E. Pérez-Payá, C. T. Dooley, C Pinilla, R. A. Houghten, Trends Anal. Chem. 14, 83 (1995).

94. K. W. Hahn, W. A. Klis, J. M. Stewart, Science 248 , 1544 (1990); M. J. Corey, E. Hallakova, K. Pugh, J. M. Stewart, Appl. Biochem. Biotechnol. 47, 199 (1994)

95. K. T. O'Neil, R. H. Hoess, W. F. DeGrado, Science 249, 774 (1990).

96. Abbreviations for the amino acid residues are: $A$ Ala; C, Cys; D, Asp; E, Glu; F, Phe; G, Gly; H, His; I lle; K, Lys; L, Leu; M, Met; N, Asn; P, Pro; Q, Gln; R, Arg; S, Ser; T, Thr; V, Val; W, Trp; and Y, Tyr.
97. T. E. Ellenberger, C. J. Brandl, K. Struhl, S. C. Harrison, Cell 71, 1223 (1992)

98. D. E. Fisher, L. A. Parent, P. A. Sharp, ibid. 72, 467 (1993).

99. A. R. Ferré-D'Amaré, P. Pognonec, R. G. Roeder, S. K. Burley, EMBO J. 13, 180 (1994)

100. J. R. Desjarlais and J. M. Berg, Proc. Natl. Acad. Sci. U.S.A. 90, 2256 (1993)

101. E. J. Rebar and C. O. Pabo, Science 263, 671 (1994); A. C. Jamieson, S.-H. Kim, J. A. Wells, Biochemistry 33, 5689 (1994); Y. Choo and A. Klug, Proc. Natl. Acad. Sci. U.S.A. 91, 11168 (1994); ibid., p. 11163; H. Wu, W.-P. Yang, C. F. Barbas III, ibid. 92, 344 (1995)

102. Y. Choo, I. Sánchez-García, A. Klug, Nature 372, 642 (1994)

103. J. U. Bowie and R. T. Sauer, Proc. Natl. Acad. Sci.
U.S.A. 86, 2152 (1989); W. A. Lim and R. T. Sauer, J. Mol. Biol. 219, 359 (1991); C. D. Waldburger, J. F. Schildbach, R. T. Sauer, Nature Struct. Biol. 2 , 122 (1995).

104. J. W. Ponder and F. M. Richards, J. Mol. Biol. 193, 775 (1987); C. Lee and M. Levitt, Nature 352, 448 (1991); H. W. Hellinga and F. M. Richards, Proc Natl. Acad Sci U.S.A. 91, 5803 (1994); J. R. Desjarlais and T. M. Handel, Protein Sci., in press.

105. V. Muñoz and L. Serrano, Proteins 20, 301 (1994)

106. A. Wada, Adv. Biophys. 9, 1 (1976); R. P. Sheridan and L. C. Allen, Biophys. Chem. 11, 133 (1980); W. G. J. Hol, Prog. Biophys. Mol. Biol. 45, 149 (1985)

107. P. J. Kraulis, J. Appl. Crystallogr. 24, 946 (1991).

108. Supported by a grant from the Office of Naval Research.

\title{
Geometry of a Black Hole Collision
}

\section{Richard A. Matzner, ${ }^{\star}$ H. E. Seidel, Stuart L. Shapiro, L. Smarr, W.-M. Suen, Saul A. Teukolsky, J. Winicour}

The Binary Black Hole Alliance was formed to study the collision of black holes and the resulting gravitational radiation by computationally solving Einstein's equations for general relativity. The location of the black hole surface in a head-on collision has been determined in detail and is described here. The geometrical features that emerge are presented along with an analysis and explanation in terms of the spacetime curvature inherent in the strongly gravitating black hole region. This curvature plays a direct, important, and analytically explicable role in the formation and evolution of the event horizon associated with the surfaces of the black holes.

Black holes are small (a black hole of a million solar masses would be only as large as the sun), distant objects that have yet to be observed directly. But definite predictions about them come from detailed studies of solutions of Einstein's equations of general relativity. Fortunately, analytical methods (1) are powerful enough to solve these equations for a single, stationary black hole. However, this is not true for dynamically interacting black holes, believed to underlie some of the most dramatic phenomena in our universe.

The two-body problem in general relativity is still unsolved and is the subject of a National Science Foundation High-Performance Computing and Communications Grand Challenge project, termed the Binary

R. A. Matzner is professor of physics and director of the Center for Relativity, University of Texas, Austin, TX 78712 , USA. H. E. Seidel is senior research scientist at the National Center for Super Computing Applications (NCSA) and visiting associate professor of physics at the University of Illinois, Urbana, IL 61801, USA. S. L. Shapiro and S. A. Teukolsky are professors of astronomy and physics at Cornell University, Ithaca, NY 14853, USA. L. Smarr is director of NCSA and professor of astronomy and physics at the University of Illinois, Urbana, IL 61801, USA. W.-M. Suen is associate professor of physics at Washington University, St. Louis, MO 63130, USA. J. Winicour is professor of physics and astronomy at the University of Pittsburgh, Pittsburgh, PA 15260, USA

*To whom correspondence should be addressed.
Black Hole Alliance. The members of the Alliance include principal investigators at eight universities as well as a number of associates, collaborators, students, and postdoctoral fellows. The focus is to solve the problem as formulated topologically by Einstein and Rosen (2) in 1935 and as formulated numerically by DeWitt and Misner (3) in 1957. Solution of this 60 -year-old problem will require the teraflop supercomputers of the late 1990s.

Supercomputers have advanced in speed by over 50,000 times since Hahn and Lindquist (4) made the first numerical attack on the problem 30 years ago. Nearly 20 years ago, Smarr and Eppley (5) obtained the first numerical solution of the head-on collision of two black holes of equal mass. They determined that the black holes did coalesce, radiating gravitational waves with energy of approximately $10^{-3} \mathrm{Mc}^{2}$, where $\mathrm{M}$ is the mass of the system and $c$ is the speed of light. The gravitational waveform was similar to the damped vibrations ("ringing modes") familiar from perturbation calculations of black holes (6). However, numerical instabilities prevented those early calculations from being used to determine the details of the coalescence, which we report here. 
The Alliance is committed to a 5-year investigation on the full inspiral and merger of two black holes orbiting about each other. This fully three-dimensional problem is vastly more complex than the head-on collision treated in this article. Nonetheless, we expect the full problem to bear many similarities. The Alliance's solutions will be very important to the LIGO gravitational wave observatory (7), which is a system of large Michelson interferometer detectors of gravitational radiation presently under construction in the United States. LIGO is part of a global network, including the FrenchItalian VIRGO detector under construction near Pisa and other detectors in various stages of planning.

Einstein's equations are a set of nonlinear, coupled partial differential equations in space and time. The numerical solution for generic configurations of black holes is at the edge of possibility by using recent advances in computer hardware and algorithms. We will not describe the details of the numerical computations here. Rather, we will present one of the first fruits of this collaborative effort: locating the precise surfaces of black holes in axisymmetric configurations as they collide, and mapping out their geometric structure.

\section{Black Holes}

John Wheeler (8) coined the insightful and provocative name "black holes" in the context of general relativity, Einstein's theory of gravity. However, the major properties of black holes can be understood in terms of Newtonian physics, together with the relativistic principle that nothing can travel faster than the speed of light. A black hole is an "object" with an escape velocity equal

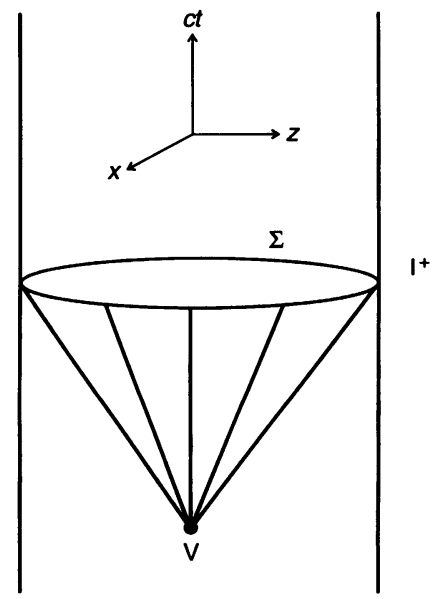

Fig. 1. Photons emanating from a flashbulb generate a compactified light "cone" in flat spacetime. V, vertex (origin of the light rays); $\mathrm{I}^{+}$, infinity; $\Sigma$, circle representing the last time slice at infinity through the expanding sphere of light radiating from $\mathrm{V}$; $c$, speed of light; $t$, time. to the speed of light (the escape velocity $v_{\text {esc }}$ is the minimum velocity required to send an object to infinity). Long ago Laplace (9) noted that the escape velocity from the gravitational pull of a spherical star of mass $M$ and radius $R$ is

$$
v_{\mathrm{esc}}=\sqrt{\frac{2 \mathrm{GM}}{R}}
$$

where $G$ is Newton's gravitational constant. Adding mass to the star (increasing $M$ ) or compressing the star (decreasing $R$ ) increases $v_{\text {esc }}$. When the escape velocity exceeds $c$, even light cannot escape and the star becomes a black hole. (For any known kind of matter, the gravitational field at this stage is so strong that the star inevitably collapses to an infinite density singularity.)

The required radius $R_{\mathrm{BH}}$ for a black hole of mass $M$ follows from Eq. 1, with $v_{\text {esc }}$ set equal to $c$ :

$$
R_{\mathrm{BH}}=\frac{2 \mathrm{GM}}{c^{2}}
$$

For a solar-mass black hole, $M \sim 2 \times 10^{33}$ $\mathrm{g}$ and $R_{\mathrm{BH}} \sim 3 \mathrm{~km}$. Remarkably, Eq. 2 holds even in general relativity, which describes gravitation by curvature of space and time thought of as one entity: spacetime. A black hole is a region where curvature traps light from escaping to infinity.

To portray this idea, we use spacetime diagrams in which two spatial dimensions are drawn, as well as the evolution in time (depicted as the vertical direction). The spacetime trajectories of the photons emanating from a flashbulb are shown in Fig. 1. To get a useful picture, we must use the same units on all the axes. Thus, we plot time multiplied by $c$ on the vertical axis. Then the path of a light ray, satisfying $d$ (distance)/ $d t=c$, makes an angle of $45^{\circ}$ with respect to the time axis. The third spatial direction $(y)$ is suppressed in the drawing in order to provide an intelligible perspective. Light from the flash travels outward in a spherical shell. (At any one time, a $t=$ constant slice produces a circle in our diagram, our twodimensional representation of a sphere.) To view the entire spacetime, we "compactify" it in the lightlike direction of the radiation travel, by rescaling the distance so that points at infinity, denoted by $\mathrm{I}^{+}$, are mapped to a finite location (10). With such a rescaling, the picture of the expanding shell of light has a finite outer boundary, corresponding to the last spherical time slice at infinity. The essential advantage gained here is that we can represent this last sphere by the circle $\Sigma$ in our finite-sized diagram. We will use this picture of a light "cone" in flat spacetime (spacetime with no gravitational field) to explain the curved spacetime geometry for the case of a head-on collision of two black holes.
Now consider a spherical star cluster that collapses to a black hole. Figure 2 is the spacetime diagram. The infalling stars eventually produce a region at the center where $v_{\text {esc }}=c$. As more stars fall in, this region grows. Finally, after all the stars fall in, the size of this region remains constant. The stars eventually collapse to an infinite density singularity indicated by the jagged line. The black hole first forms at the center, and as it grows, it surrounds the singularity, in accord with the hypothesis of cosmic censorship that states that singularities must not be visible to distant observers. The evolution of the surface of the black hole traces out a curved version of a light cone, indicated in Fig. 2 by $\mathrm{H}$.

Except for its curvature, $\mathrm{H}$ is analogous to the light cone in Fig. 1. It forms at $t=t_{\mathrm{B}}$ (B for "birth") in the diagram, where light rays from the center of the cluster just miss escaping to infinity. These light rays provide the skeletal structure of the black hole analogous to the way straight lines rule (generate) a cone in Euclidean geometry. As these rays diverge outward from the center, the gravitational attraction of the infalling matter distorts the spacetime so that they do not expand indefinitely but "hover" at the final black hole radius. At late times they therefore define a nondiverging (and nonconverging) parallel cylinder (topologically $S^{2} \times R^{1}$, where $S^{2}$ is the sphere and $R^{1}$ is the line), which is generated by the rays.

Imagine repeatedly setting off a flash at the center. Before the black hole forms, the light flashes travel out to infinity. The surface of the black hole is determined by that particular light cone that just misses escaping to infinity. This is a subtle concept that involves the complete future history of the system. One must first find the special sphere of light rays that is hovering at late times and then trace it back to find its

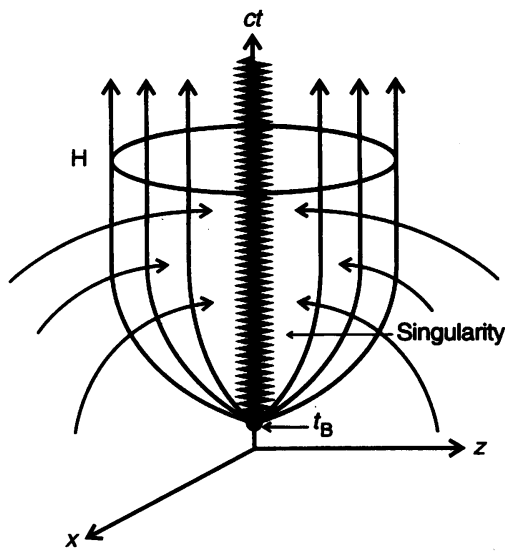

Fig. 2. Spacetime diagram for a spherical collapse to a black hole. $H$, trace of the evolution of the black hole surface or horizon; $t_{\mathrm{B}}$, time at which the black hole was "born." 
birth. To appreciate the reverse time order necessary for this construction, imagine a diagram like Fig. 2 but with an additional hollow spherical shell of matter falling in at late times. The additional gravitational attraction from this shell would be sufficient to refocus to a point the nondiverging portion of the cone drawn in Fig. 2. Indeed, in that case, a cone that emerged from a slightly earlier flash, and that would otherwise expand to infinity, would now be deflected inward to exactly zero divergence at late times and would thus form the actual black hole surface.

The final equilibrium state formed after matter and energy stop falling into the hole is well understood. There are theorems that state that when the black hole eventually settles down to a stationary configuration, its gravitational field has the analytic form of a Kerr black hole which is uniquely determined by its mass and angular momen-

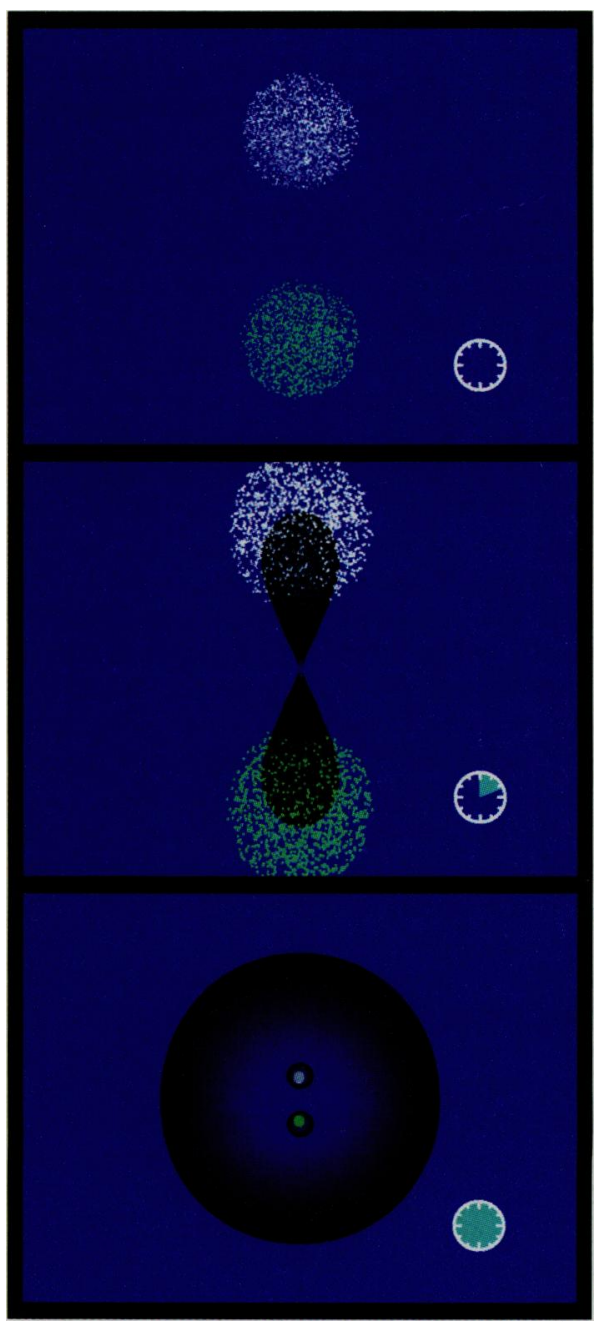

Fig. 3. Snapshots of the collision and merger of two black holes formed by the collapse of two balls of particles. The spatial location of the event horizon is shown in black. The clock in each frame shows the fraction of time elapsed during the simulation. tum. They are called "no hair" theorems to emphasize the simplicity of the black hole equilibrium state. (Electric charge is also allowed but is irrelevant for astrophysically realistic black holes.)

Because light cannot escape the black hole surface, events that occur inside cannot be seen from outside. Hence, the name black hole event horizon is used to denote the evolution of the black hole surface. It is a horizon because we cannot see beyond it. In our computations to be described below, we use the fact that the black hole state is known at late times to find the event horizon at late times. Then our methods integrate back into the past to determine the horizon structure during the earlier dynamical part of the evolution.

General relativity allows not only black holes formed by matter collapse but also black holes that are stable topological structures in spacetime and need have no matter content at all. Such black holes do have mass, and the nonlinearity of their gravitational field creates curvature that holds them together. We will return to such "eternal" black holes below (11-13).

Referring to Fig. 2, we can verify several key properties that apply to event horizons in general: (i) The horizon is generated by light rays passing through each point in the horizon, and (assuming cosmic censorship) these generators continue along the horizon forever into the future. (ii) The horizon begins where these generators meet in the past. (iii) The cross-sectional area of the horizon monotonically increases and approaches a constant at late time. (For a single spherical black hole, the area approaches the value $4 \pi R_{\mathrm{BH}}{ }^{2}$.)

The collision of two black holes cannot be treated analytically and requires fullfledged computation. We have simulated the head-on collision of nonrotating black holes of equal mass to form a single black hole (14). In this simulation, each black hole is formed from the collapse of a spherical ball of noncolliding particles, analogous to the stars in a spherical galaxy. Each ball collapses because the particles are chosen to have no initial motion. Thus, there is no centrifugal force to balance the inward pull of gravity. To mimic a collision, we begin the simulation with the two clusters well separated but headed toward each other with a velocity of 0.15 c. This initial configuration is shown in the first frame of Fig. 3 (the initial separation is about five times the radius of the final black hole). The two clusters then fall toward each other while individually collapsing to form black holes. The individual black holes originate at the center of each cluster and then grow outward. The second frame of Fig. 3 depicts the instant at which these two tidally distorted black holes first touch. The black holes then merge and in the process convert $\sim 3 \times 10^{-4}$ of the system's mass into energy in the form of gravitational waves radiating outward. The last frame of Fig. 3 shows the end of the simulation, with a single black hole encompassing all of the matter. The final black hole is settling into a spherical equilibrium state.

Figure 3 provides a picture, in ordinary three-dimensional space, of the black hole formation and merger process at three instants of time. The complete numerical evolution constructs these spatial pictures in an almost continuous way, like the frames of a movie. From these spatial pictures, we can reconstruct a four-dimensional spacetime description of the evolution. In a spacetime diagram, each frame of the type shown in Fig. 3 would correspond to a horizontal slice, representing all of space at a given instant of time. We have mapped out the location of the event horizon in this computed spacetime by numerically propagating light rays [see (15), especially figure 3].

Figure 4 is a spacetime picture showing some of these light rays superimposed on the resulting event horizon. It was constructed by propagating light rays backward in time from the surface of the final equi-

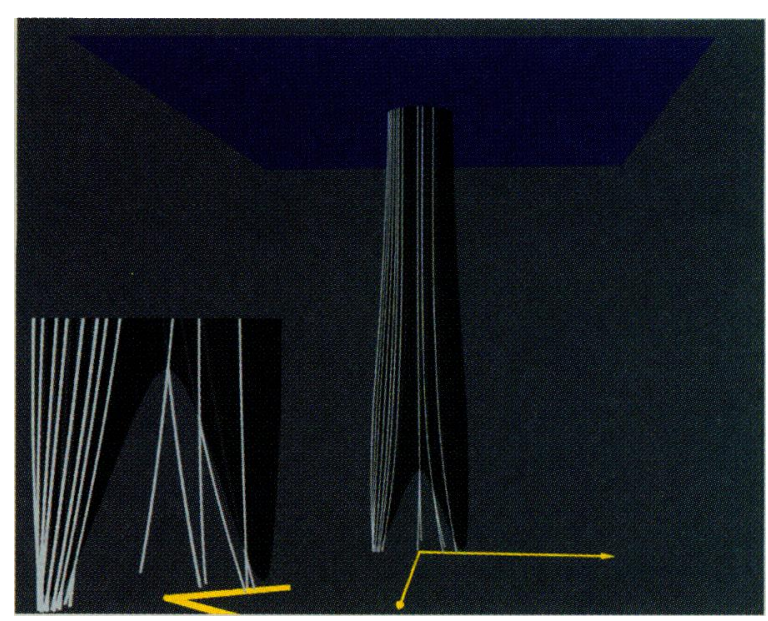

Fig. 4. Computational construction of some of the light rays generating the horizon for the case shown in Fig. 3. The collision axis goes from left to right, and the time axis is vertical. The inset zooms in on the caustic and crossover structure at the birth of the horizon. 
librium black hole with the ideas in (16, 17). The blue slice at the top of Fig. 4 corresponds to the end of the simulation, the final frame of Fig. 3. The picture represents a calculation of the "pair of pants" description of the event horizon as sketched by Hawking and Ellis [(18), figure 60] or Misner, Thorne, and Wheeler [(19), figure $34.6]$ more than 20 years ago. We see by tracing the rays backward in time that some of them cross each other and leave the horizon. The simulation helps reveal the important role of these crossovers as the initial events where the horizon forms. The inset shows a closer view of the beginning of the horizon and how the rays behave near those events.

The crossovers are the earliest points along the rays that lie on the horizon. Before crossing, these rays travel in a region of spacetime to the past of the horizon that is visible to a distant observer. This is clear from the light cone of Fig. 1: any ray continued backward through the vertex $\mathrm{V}$, where it intersects other rays, enters the spacetime region to the past of $\mathrm{V}$, which is visible to distant observers at $\mathrm{I}^{+}$at times before $\Sigma$. Crossovers are ubiquitous features of black hole interactions. The attractive nature of gravity causes even a beam that initially has perfectly parallel rays to bend and self-intersect, analogous to the intersection of two flashlight beams from separate directions. In Fig. 4, a line of such crossover points extends from the "crotch" on the "pair of pants" down along each inside trouser "seam," around each bottom, and a small distance up each outside "seam." At the endpoints of the line of crossovers, slightly up the outside of each "leg," are caustics, analogous to a focal point where a single flashlight beam would converge to infinite intensity (if it were not for diffraction). In the spherically symmetric case, the beam emerging from a point caustic would, if gravity were weak, trace out the light cone of Fig. 1 or, if gravity were sufficiently strong, trace out the horizon for the single black hole of Fig. 2. The tidal effects of the colliding black holes shift the location of the caustics and lead to the line of crossovers. We will see below how this comes about.

One feature of Fig. 4 deserves comment. The figure makes it appear that the area of the horizon decreases at late times, contrary to property (iii) above. In fact, a careful check of the computed spacetime shows that the horizon area increases asymptotically to a constant value at late times. The apparent decrease is an artifact of the coordinates chosen to represent the horizon in Fig. 4. Figure 10, which is for a different simulation and is fully discussed in the section entitled "Vacuum Black Hole Collisions," is a visualization in which this effect has been rigorously controlled (20).

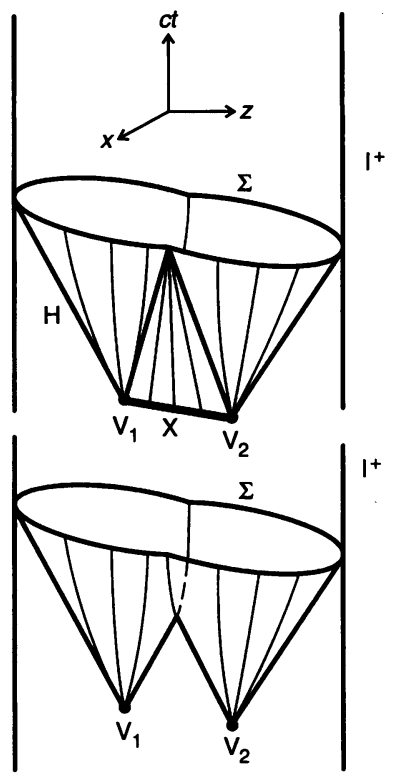

Fig. 5. The lower diagram illustrates colliding light cones in a flat spacetime. The upper diagram illustrates the event horizon corresponding to the surface $\Sigma$ at infinity $\mathrm{I}^{+}$. The compactification distorts spatial directions so that the $(c t, x, z)$ "compass" is only schematic. In particular, the $+x$ direction ray from $V_{1}$ intersects the $+x$ direction ray from $V_{2}$ at the kink in $\Sigma$. In the diagrams, these rays appear to have a spurious $z$-motion, which is the price we pay for compactification. Similar comments apply to Figs. 6 and 9. X, crossover line.

\section{Colliding Light Cones}

Consider the collision of two black holes in terms of the spacetime picture of the collision of two light cones depicted in the lower diagram of Fig. 5. In this compactified picture, the vertices $V_{1}$ and $V_{2}$ of the light cones are separated along the $z$ axis, about which the collision has rotational symmetry. This axisymmetry makes it possible for a three-dimensional $(t, x, z)$ picture to represent all aspects of the collision. Each point on the $x$ axis corresponds to a circle of axisymmetry in the $x, y$ plane, centered about the $z$ axis. In the picture, points with the same $t$ and $z$ but with values $\pm x$ lie on the same circle, and the circle reduces to a single point on the $z$ axis where $x=0$.

Each light cone intersects infinity in a sphere. But to discuss a "sphere at late times" that resembles the late-time spherical surface of a black hole, we restrict attention to the two "outside" hemispheres in which the light rays from one vertex reach infinity without hitting rays from the other vertex. These two hemispheres join together to form $\Sigma$, the closed two-dimensional surface at infinity indicated in Fig. 5. The surface $\Sigma$ is kinked where the two hemispheres join in a circle in the $x, y$ plane. Our figure shows two kinks (corresponding to the two intersections of that circle with the

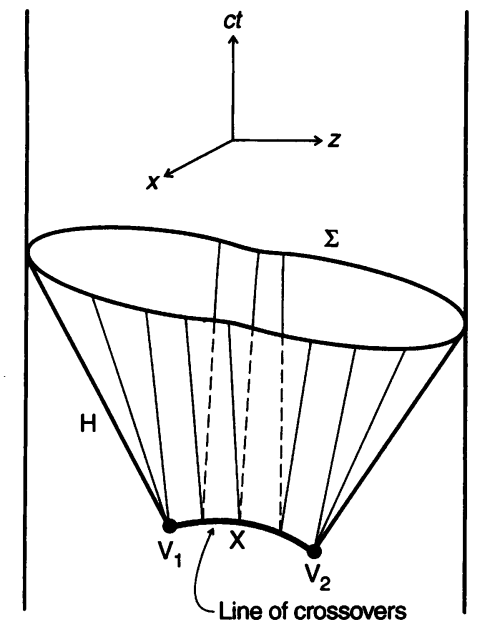

Fig. 6. Deformation of the crossover line in Fig. 5 due to spacetime curvature.

$x$ axis). One of these kinks corresponds to the two light rays, one from each vertex, traveling in the positive $x$ direction. Because these two rays are parallel, they meet at infinity. The other kink corresponds to the analogous rays traveling in the negative $x$ direction from the vertices. [The radial compactification distorts the apparent direction of the rays so that the ray in the $+x$ direction from each vertex appears to have a motion in the $z$ direction. We must draw them this way so that the parallel rays eventually intersect at $\mathrm{I}^{+}$, in this case at the kink in $\Sigma$. This means that we view the $(c t, x, z)$ "compass" in Fig. 5 as only schematic.]

The collision takes place between the hemisphere of rays from each vertex that head toward each other. The ray in the positive $z$ direction from $V_{1}$ collides first with the ray in the negative $z$ direction from $V_{2}$. The entire set of colliding rays is indicated in the figure by the parabola, which is the conic section formed by the intersection of the light cones with the $x, t$ plane located midway between them.

In this flat spacetime model, the parabola of collision points is the analog of the strong geometrical interaction occurring in the collision of two black holes. The analog of the horizon $\mathrm{H}$ is the boundary of the region visible to observers at infinity before the time corresponding to the surface $\Sigma$. The analog of an observer in a black hole spacetime is an observer outside the light cone stretching back from $\Sigma$. We shall call that structure $\mathrm{H}$ in this case also.

To construct $\mathrm{H}$, we trace back all of the light rays reaching $\Sigma$ until they meet one of the other rays in either a crossover or a caustic. Obviously, the rays from one outside hemisphere converge to $V_{1}$ and those from the other outside hemisphere converge to $\mathrm{V}_{2}$.

But what about the rays traced back from the kinks on $\Sigma$ where the hemispheres join? 


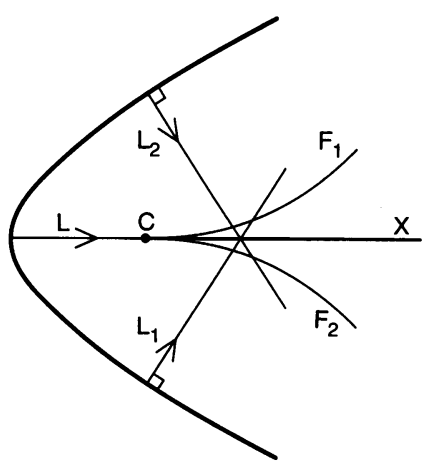

Fig. 7. Spatial picture of a generic cusp caustic (C) produced by an aspherical beam. $F$, fold line; $L$, light ray.

Two of these are the rays from $V_{1}$ and $V_{2}$ that travel in the positive $x$ direction. These parallel rays intersect at infinity. In fact, all parallel rays in the positive $x$ direction starting from points along the $z$ axis between $V_{1}$ and $V_{2}$ have the same "endpoint" at infinity, the kink at the end of the parabolic arc formed by the colliding outgoing rays. In the backward time direction, each of these rays intersects, at $x=0$, the opposite ray traveling in the negative $x$ direction from the opposite kink. From these clues, we see that $\mathrm{H}$ corresponds to the two half-cones from $V_{1}$ and $V_{2}$ together with the two sets of parallel rays sweeping out in the $\pm x$ direction as illustrated in the upper diagram of Fig. 5. These rays originate at points of $\mathrm{H}$ along the spatial line on the $z$ axis connecting $V_{1}$ and $V_{2}$. This part of the $z$ axis is the crossover line where rays from $\pm x$ direction intersect. Note also the surprising result that the parabola formed by the colliding rays is to the future of $\mathrm{H}$ and therefore invisible to observers "outside the horizon."

\section{Deforming the Picture}

Our picture is beginning to resemble the horizon formed in the simulated collision between two black holes. To make it more realistic, the gravitational effect of spacetime curvature must be included. The strong gravitational field near the holes causes light rays to bend inward toward one another. Tracing the rays generating $\mathrm{H}$ backward from infinity, this effect causes them to intersect "sooner" along a curved spatial crossover line, as depicted in Fig. 6. In this figure, we have also smoothed out the kinks on $\Sigma$, which has a similar effect as curvature to cause the crossover line $\mathrm{X}$ where $\mathrm{H}$ originates to bulge upward. $\mathrm{X}$ corresponds to a seam on the inside pant legs in the trouser picture of the black hole collision; the shape is bowlegged, and the profile corresponds to a smooth spatial line with no special sharpness at the midpoint of the seam where the legs join.

To complete the picture, another subtle

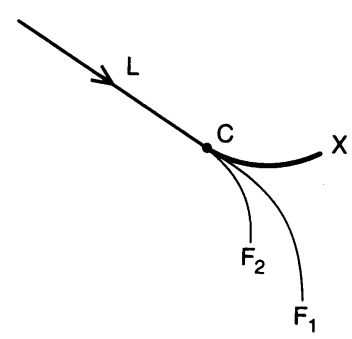

Fig. 8. Spacetime version of Fig. 7 , with time directed vertically.

effect of curvature must be included. The vertices of the light cones in Fig. 6 are caustic points corresponding to the focus of a finite solid angle of rays. These point caustics arise from the spherical symmetry of the light cones in our flat spacetime model. In reality, point caustics are highly unlikely to occur in a generic system. Any slight perturbation of spherical symmetry destroys the perfect focus in an unstable, unpredictable manner. Such results have been made rigorous by using the methods of catastrophe theory (21) and nonlinear dynamics (22). The spatial geometry of the ray structure of the elementary stable caustics has received a great deal of attention because of their omnipresence in classical and diffraction optics (23).

In the presence of rotational symmetry about an axis, as in our simulations, the only stable caustics consist of cusps and folds. Figure 7 illustrates a spatial picture of a typical axisymmetric wavefront converging to a caustic, with the circles of axisymmetry suppressed. The light ray $L$ encounters a cusp caustic at the point $C$, but light rays $L_{1}$ just below $L$ do not focus at $C$ but along the fold line $\mathrm{F}_{1}$. Similarly, light rays $\mathrm{L}_{2}$ focus along the fold line $\mathrm{F}_{2}$. Of special importance to the structure of horizons, the rays $L_{1}$ and $L_{2}$ intersect along a crossover line $\mathrm{X}$ before they reach their respective fold lines. Hence, we expect the point caustics of our flat space model to be replaced by cusp caustics, connected by a crossover line.

The major theoretical investigations of caustics have typically dealt with steady bright patterns, which result from the timeaveraged appearance of an optical frequency beam. The spacetime geometry has received relatively little attention, except in general relativity. The pioneering studies of Penrose (24), which led to the first proof that singularities result from gravitational collapse, were based on the spacetime properties of caustics. Many details of the spacetime geometry of the elementary stable caustics have now been worked out (25).

Figure 8 is the spacetime version of Fig. 7 and exhibits some of those details. The ray $\mathrm{L}$ traced backward in time along the horizon encounters a cusp point $\mathrm{C}$. From the spacetime event $\mathrm{C}$, $\mathrm{L}$ continues on into the past, but for simplicity we suppress that here, because the portion of $L$ to the past of $\mathrm{C}$ is not on the horizon but in the visible region of spacetime. The horizon begins where its generating rays meet, either at caustics or at crossover points. The fold lines $F_{1}$ and $F_{2}$ are also not on the horizon because the rays $\mathrm{L}_{1}$ and $\mathrm{L}_{2}$ meet (traveling backward) along the crossover line $\mathrm{X}$ before they meet $F_{1}$ or $F_{2}$. Only the cusp $C$ at the tip of the crossover line is on the horizon and thus not visible to distant observers.

The entire crossover line $\mathrm{X}$ is also on the horizon; it is where the bulk of the generators originate. Crossover lines, which arise when two separate beams of rays intersect, must be spacelike. This means that the lines cannot be traced by light rays or particles moving at less than $c$. Their detailed properties have not been studied as extensively as caustics, where a single beam focuses. Our computational study of the cusp caustic reveals the surprising result that the crossover line, although spacelike at each point, becomes asymptotically lightlike as it approaches the cusp $\mathrm{C}$. As a result, $\mathrm{X}$ joins smoothly onto the light ray $\mathrm{L}$, as shown in Fig. 8. This is the final clue necessary to deform Fig. 6 into the horizon found in the collision between black holes.

\section{Putting the Pieces Together}

The qualitative features of the stable caustics are preserved by perturbations, whether due to the refractive properties of a medium or to the gravitational curvature of spacetime. Even more relevant to the simulation of black holes, they are insensitive to small numerical error. Thus, in the axisymmetric case of a head-on collision, one expects to find cusps on the horizon. However, the stability is only a local property that describes the generic behavior in the neighborhood of the cusp. Pieces of the horizon can be expected to resemble Fig. 8 , but the pieces must be put together. The way to do this is suggested by a comparison of Figs. 6 and 8 . By replacing the unstable feature of Fig. 6, the point vertices $V_{1}$ and $V_{2}$, each by the cusp of Fig. 8, we obtain the stable horizon structure shown in Fig. 9. Figure 9 supplies the "key" to the computed black hole structure in Fig. 4. It shows a cusp C on the outside of each "trouser leg." The cusps are connected by a spatial crossover line X, which forms a seam on the trousers. The seam joins smoothly with the two light rays, analogs of $L$ in Fig. 8, emanating from the cusps (26). Those rays are exceptional. The other light rays emanate from $\mathrm{X}$ in pairs (the circles of axisymmetry being suppressed), one up the front of the trousers and one up the back. The final black hole equilibrium state long after the collision is a sphere, represented by a circle (and $\Sigma$ is 
almost circular) in our figure. This provides a consistent theoretical model for our computations.

A final point. The crossover line drawn in Fig. 9 is actually a caustic in the exactly axisymmetric case, because it is the focal line of a circle of rays in the $x, y$ plane. However, under a perturbation away from exact axisymmetry there would be defocusing, and this line would broaden into a two-dimensional crossover surface.

\section{Vacuum Black Hole Collisions}

We turn now to vacuum black holes, often called "eternal" black holes because they are not formed from matter collapse but are permanent features of solutions of the nonlinear Einstein equations. At $t=t_{\text {initial }}$, we choose a system consisting of two nonrotating black holes poised near one another. The field data are momentarily stationary (the field momenta vanish and $t_{\text {initial }}$ is a moment of time symmetry of the fields, though as we shall see, the horizon is not time symmetric) (27). We have evolved this system $(16,11$ $13,28)$ into the future from $t_{\text {initial }}$ for a time $\sim 150 \mathrm{GM} / \mathrm{c}^{3}$, and backward in time for $\sim 8$ $\mathrm{GM} / \mathrm{c}^{3}$ (where $M$ is the mass of the final black hole) (Fig. 10). For a solar mass black hole, these times are less than $10^{-3} \mathrm{~s}$, indicating the violent nature of the merger.

At late times, the horizon oscillates with decaying amplitude around a spherical shape $(12,16,17)$. When disturbed, a black hole will vibrate and emit gravitational waves with a characteristic frequency determined by its mass and spin, just as a bell emits sound waves when struck by a mallet. As these ringing modes subside, the hole settles down to the known equilibrium solution, which provides us with the black hole surface at late times. We then integrate back-

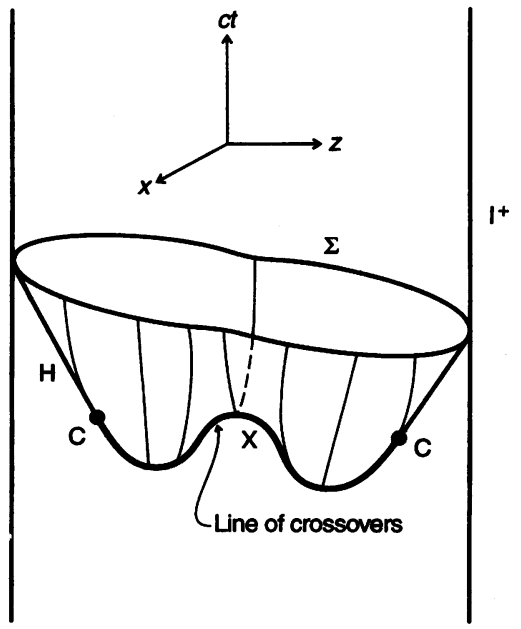

Fig. 9. Key for Fig. 4: The horizon formed by the merger of two black holes. The base contains two versions of the cusp in Fig. 8 joined by a common crossover line $X$. ward in time $(16,17)$ to locate the surface at earlier times. Figure 10 shows the horizon structure in the most interesting epoch, starting from $5 \mathrm{GM} / \mathrm{c}^{3}$ in the past of beginning of coalescence up to $20 \mathrm{GM} / \mathrm{c}^{3}$ in the future. Figure 10 differs from Fig. 4 in two important ways. First, the black holes in Fig. 4 are "born" and the horizon's origin can be studied, as discussed above, whereas the black holes in Fig. 10 exist eternally. Second, Fig. 4 gives a coordinate description of the horizon, whereas Fig. 10 gives a more intrinsic visualization of the horizon geometry in terms of the time evolution of its polar circumference. This reveals the monotonic increase and asymptotic constancy of the area, consistent with the black hole theorems.

Unlike the matter-filled collapse, the trouser legs in Fig. 10 do not have cusps but continue into the past from our diagram. We do find a crossover line along the inside trouser seam, exactly analogous to that in Fig. 4 for our matter simulation. Although spacetime is symmetric in time about $t_{\text {initial, }}$, the horizon itself is not time-symmetric rays but expands monotonically into the future. This expansion of the actual rays tracing the horizon is clearly visible at $t_{\text {initial }}$ in Fig. 10.

From the time symmetry of the system, one might have naively (and incorrectly) concluded that its past evolution describes a time-reversed collision: radiation impinging on a single black hole, blowing it into two holes that come to a momentarily stationary pause at the "initial" time, with radiation then emitted as the holes merge. Such expectations of the dynamics turn out to be wrong. Except in a completely stationary case, the horizon cannot behave symmetrically about $t_{\text {initial }}$. This is an obvious conclusion from two theorems by Hawking: The area of a black hole always increases (29) [see property (iii) above], and black holes cannot bifurcate (30). Generically, an event horizon is not time symmetric.

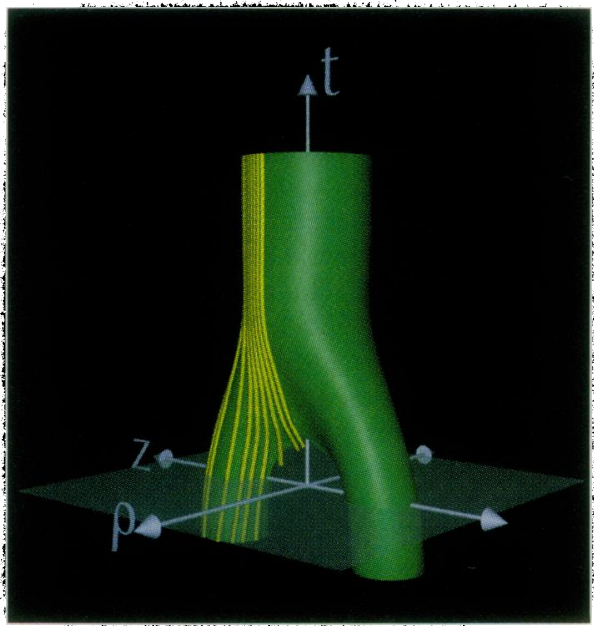

Fig. 10. Computer simulation of the collision between two vacuum black holes.

\section{Conclusion}

Before having computational tools for studying the collision and merger of two black holes, we had to rely on sketches and intelligent guesses to describe the interaction. Now the numerical simulations have revealed new qualitative features of the collision and have pointed the way to simple analytic models that reveal the underlying physics. Specifically, the computations have led to the discovery of the exact nature of the crossover line and its caustic endpoints at the formation of the horizon.

An even more formidable challenge will be to track the coalescence and merger of two black holes in a circular orbit as they spiral together because of the emission of gravitational waves. This scenario is astrophysically more realistic than the head-on collision considered here. However, the total lack of symmetry will require sophisticated new algorithms and will tax the most powerful computers. Work is in progress to meet this next challenge.

\section{REFERENCES AND NOTES}

1. K. Schwarzschild, Sitzungsber. Dtsch. Akad. Wiss Berlin, KI. Math. Phys. Tech. 1916, 189 (1916); R. P. Kerr, Phys. Rev. Lett. 11, 237 (1963).

2. A. Einstein and N. Rosen, Phys. Rev. 48, 73 (1935)

3. See, for example, L. Smarr, in Quantum Theory of Gravity, S. Christensen, Ed. (Hilger, Bristol, 1984), p. 14.

4. S. Hahn and R. W. Lindquist, Ann. Phys. NY 29, 304 (1964).

5. L. Smarr, Sources of Gravitational Radiation (Cambridge Univ. Press, Cambridge, 1979), p. 245.

6. See, for example, S. Chandrasekhar, The Mathe matical Theory of Black Holes (Oxford Univ. Press, Oxford, 1983).

7. A. Abramovici et al., Science 256, 325 (1992).

8. J. A. Wheeler, Am. Sci. 56, 1 (1968).

9. P. S. Laplace, Le Système du Monde (Paris, 1795), vol. II [Engl. transl. (Flint, London, 1809)].

10. R. Penrose, Phys. Rev. Lett. 10, 66 (1963).

11. P. Anninos, D. Hobill, E. Seidel, L. Smarr, W.-M. Suen, ibid. 71, 2851 (1993).

12. P. Anninos et al., Phys. Rev. D 50, 3801 (1994).

13. P. Anninos, D. Hobill, E. Seidel, L. Smarr, W.-M. Suen, ibid., in press.

14. S. Shapiro and S. Teukolsky, ibid. 45, 2739 (1992).

15. S. Hughes et al., ibid. 49, 4004 (1994).

16. P. Anninos et al., Phys. Rev. Lett. 74, 630 (1995).

17. J. Libson et al., in preparation.

18. S. W. Hawking and G. F. R. Ellis, The Large Scale Structure of Spacetime (Cambridge Univ. Press, Cambridge, 1973)

19. C. W. Misner, K. S. Thome, J. A. Wheeler, Gravitation (Freeman, San Francisco, 1973).

20. General relativity allows a wide choice of the kind of simultaneity "slices" for the description of spacetime. Both the simulation shown in Figs. 3 and 4 and the simulation of Fig. 10 use times-for instance, that shown on the clock in Fig. 3-which are variants of the so-called maximal sticing. [See, for example, L. Smarr and J. W. York Jr., Phys. Rev. D 17, 1945 (1978); ibid., p. 2529.]

21. R. Thom, Stabilité Structurelle et Morphogenèse (Benjamin, Reading, MA, 1972) [Structural Stability and Morphogenesis, English translation (Benjamin, Reading, MA , 1975)]

22. V. I. Arnold, Matematicheskje Metody Klassichesko Mekhaniki (Nauka, Moscow, 1974) [Mathematica Methods of Classical Mechanics, English translation (Springer-Verlag, New York, 1978)].

23. M. V. Berry and C. Upstill, Prog. Opt. XVIII, 256 (1980). 
24. R. Penrose, (1963) reprinted in Gen. Rel. Grav. 12, 225 (1980).

25. H. Friedrich and J. M. Stewart, Proc. R. Soc. London Ser. A 385, 345 (1983)

26. Because the crossover line is spacelike, the "bowleggedness" of the diagram is to some extent due to our choice of coordinates. A different choice of simultaneity can change the qualitative shape but must leave invariant the smooth merger of the spacelike crossover line with the light ray lines leaving the cusps.

27. C. Misner, Ann. Phys. NY 24, 102 (1963).

28. W.-M. Suen, paper presented at Grand Challenge Meeting, National Center for Supercomputing Applications (NCSA), University of Illinois at Urbana-
Champaign, 6 to 8 November 1994 (lecture notes available from NCSA).

29. S. W. Hawking, in Black Holes, C. DeWitt and B. DeWitt, Eds. (Gordon and Breach, New York, 1973) 30. Commun. Math. Phys. 25, 152 (1972).

31. We thank D. Eardley, R. Geroch, R. Gómez, J. M. Stewart, K. Thorne, F. Tipler, and M. Visser for helpfu comments on this work. We are especially grateful to J. Massó and P. Walker for producing Fig. 10 and for contributing significantly to the analysis in this paper. We also thank M. Blanton, M. Chia, S. Hughes, C. Keeton, P. Walker, and K. Walsh for participating in the computation and production of Figs. 3 and 4 . This research was funded by the NSF Grand Challenge Grant PHY93-18152/ASC93-18152 (ARPA supple- mented) and supported by the following individual grants: by NSF grant PHY83 10083, Texas TARP. 085, and a Cray University Research Grant (R.A.M.) by NCSA and by NSF grant PHY 94-07882 (H.E.S. and L.S.); by NSF grants AST 91-19475 and PHY 94-08378 and National Aeronautics and Space Administration grant NAGW-2364 (S.L.S. and S.A.T.); by NSF grant PHY 94-04788 (W.-M.S.); and by NSF grant PHY92-08349 (J.W.). Computer time was made available by NCSA and the Pittsburgh Supercomputing Center (in part through MetaCenter grant MCA94P015), and by the Comell Center for Theory and Simulation in Science and Engineering (supported in part by NSF, IBM Corporation, New York State and the Cornell Research Institute)

\title{
Geophysics of the Pitman Fracture Zone and Pacific-Antarctic Plate Motions During the Cenozoic
}

\author{
Steven C. Cande, Carol A. Raymond, Joann Stock, \\ William F. Haxby
}

Multibeam bathymetry and magnetometer data from the Pitman fracture zone (FZ) permit construction of a plate motion history for the South Pacific over the past 65 million years. Reconstructions show that motion between the Antarctic and Bellingshausen plates was smaller than previously hypothesized and ended earlier, at chron C27 (61 million years ago). The fixed hot-spot hypothesis and published paleomagnetic data require additional motion elsewhere during the early Tertiary, either between East Antarctica and West Antarctica or between the North and South Pacific. A plate reorganization at chron C27 initiated the Pitman FZ and may have been responsible for the other right-stepping fracture zones along the ridge. An abrupt $\left(8^{\circ}\right)$ clockwise rotation in the abyssal hill fabric along the Pitman flowline near the young end of chron C3a (5.9 million years ago) dates the major change in Pacific-Antarctic relative motion in the late Neogene.

The Pacific-Antarctic Ridge is the key link in the global plate circuit tying the relative motion of the oceanic plates of the Pacific basin to the rest of the world $(1,2)$. For example, one of the more astounding consequences of rigid plate tectonics is that the accuracy of models of western North American deformation, including motion on the San Andreas fault, depends on how well magnetic anomalies and fracture zones (FZs) can be reconstructed in the far South Pacific $(3,4)$. Because key areas in the South Pacific are remote and poorly surveyed, circum-Pacific plate reconstructions have continued to have large uncertainties while uncertainties in other links in the global plate motion circuit have been progressively reduced. In this paper we describe

S. C. Cande is at the Scripps Institution of Oceanography, La Jolla, CA 92093-0215, USA. C. A. Raymond is at the Jet Propulsion Laboratory, Mail Stop 183-501, California Institute of Technology, Pasadena, CA 911098099, USA. J. Stock is at the Seismological Laboratory, Mail Stop 252-21, California Institute of Technology, Pas adena, CA 91125, USA W. F. Haxby is at LamontDoherty Earth Observatory, Palisades, NY 10964, USA. a geophysical survey of an FZ near the southernmost end of the Pacific-Antarctic ridge that enables us to greatly reduce the uncertainties in the global plate circuit.

Plate motions and global tectonics. Several fundamental questions of circum-Pacific tectonics can be addressed by a better understanding of Pacific-Antarctic plate motions. For the South Pacific, some of the most important unresolved issues involve the timing and amount of early Tertiary relative motion between East and West Antarctica and between the Lord Howe Rise and Campbell Plateau, two of the links in the plate loop tying the Pacific, Australia, and Antarctic plates together $(2,5)$. A global hot-spot reference frame also depends on the accuracy of the Pacific-Antarctic link. Atlantic and Indian Ocean hot spots, when rotated back to the Pacific, do a poor job of predicting the track of the Hawaiian hot spot $(6-8)$, raising questions of the fixity of hot spots (9). Non-Pacific paleomagnetic poles, when rotated back to the Pacific, do not agree with Pacific plate pa- leomagnetic poles. This misfit has led to the suggestion that there may be one or more missing plate boundaries between the North Pacific and East Antarctica $(10,11)$.

These unresolved questions have led to several hypotheses. Stock and Molnar (9) proposed that several puzzling aspects of early Tertiary Pacific tectonics could be explained if there was an undiscovered fossil spreading center in the Southeast Pacific that separated the region of the Antarctic plate near the Bellingshausen Basin (referred to as the Bellingshausen plate) from the part of the Antarctic plate adjacent to Marie Byrd Land (see Fig. 1). This scenario provided a better fit to magnetic anomaly data from chron $\mathrm{C} 30$ to chron $\mathrm{C} 25$ south of the Campbell Plateau; it reduced the amount of unexpected motion in the loop among the Australia, Antarctic, and Pacific plates in the early Tertiary; and it also helped to explain the failure of global reconstructions to predict the bend in the Hawaiian-Emperor chain (12).

Numerous tectonic issues can also be addressed by increasing the resolution of plate reconstructions. Several studies have proposed that there was a late Neogene change in the absolute motion of the Pacific plate, corresponding to a clockwise rotation in the separation direction of the $\mathrm{Pa}$ cific and Antarctic plates (13-16). The age of this event, however, has been difficult to establish with the pre-existing data sets; estimates of its age vary from less than 9.8 $\mathrm{Ma}$ (million years ago) (13), $5 \mathrm{Ma}$ (14), 5 to $3.2 \mathrm{Ma}(15)$ to 3.86 to $3.4 \mathrm{Ma}$ (16). Its exact age is of considerable interest because a number of late Neogene events around the Pacific, such as the onset of Pliocene compression along the San Andreas fault $(15,17)$, have been attributed to it.

Recent studies of the Pacific-Antarctic ridge have focused on interpreting satellite radar altimetry data collected by SEASAT and GEOSAT, which provide new data on the location of FZs (18-20). However, although images of the gravity field over the seafloor generated from satellite radar altimetry measurements have provided improved locations and trends of FZs and other tectonic features, without shipboard geophysical measurements in certain critical 


\section{Science}

\section{Geometry of a Black Hole Collision}

Richard A. Matzner, H. E. Seidel, Stuart L. Shapiro, L. Smarr, W.-M. Suen, Saul A. Teukolsky and J. Winicour

Science 270 (5238), 941-947.

DOI: $10.1126 /$ science.270.5238.941

ARTICLE TOOLS

REFERENCES

PERMISSIONS http://science.sciencemag.org/content/270/5238/941

This article cites 18 articles, 1 of which you can access for free http://science.sciencemag.org/content/270/5238/941\#BIBL

http://www.sciencemag.org/help/reprints-and-permissions 